\title{
An Efficient Heuristics for Minimum Time Control of Continuous Petri nets *
}

\author{
Hanife Apaydin-Özkan, ${ }^{*}$ Jorge Júlvez,${ }^{*}$ Cristian Mahulea, ${ }^{*}$ \\ Manuel Silva* \\ * Instituto de Investigación en Ingeniería de Aragon (I3A), \\ Universidad de Zaragoza, Spain (e-mails: hapaydin, julvez, cmahulea, \\ silva@unizar.es)
}

\begin{abstract}
This paper considers the problem of controlling timed continuous Petri nets under infinite server semantics. The proposed control strategy assigns piecewise constant flows to transitions in order to reach the target state. First, by using linear programming, a method driving the system from the initial to the target state through a linear trajectory is developed. Then, in order to improve the time of the trajectory, intermediate states are added by means of bilinear programming.
\end{abstract}

Keywords: Timed continuous Petri nets, minimum time control, piecewise linear trajectory

\section{INTRODUCTION}

Petri nets (PNs) are frequently used for modeling, analysis and synthesis of discrete event systems. The distributed state or marking of a $\mathrm{PN}$ is given by a vector of natural numbers which represent the number of tokens in each place. Similarly to most formalisms for discrete event systems, PNs suffer from the state explosion problem. One possible way to overcome this problem is fluidification, a classical relaxation technique.

Continuous Petri nets are a fluid approximation of classical discrete Petri nets (David and Alla (2005); Silva and Recalde (2004)). Unlike conventional PNs, a transition in a continuous Petri net can be fired in a "real" non integer quantity. This leads to continuous markings instead of discrete ones. Similarly to discrete PNs, a time delay can be associated to the firing of transitions, the resulting net is called timed continuous Petri net (contPN).

As in discrete PNs, different server semantics can be adopted for the firing of transitions. Among them the most widely used semantics are finite server and infinite server. In Mahulea et al. (2009), it is shown that infinite server semantics provides a better approximation of the steady state throughput than finite server semantics for a wide class of Petri nets under some general conditions.

A ContPN system with infinite server semantics is a subclass of Piecewise Linear Systems (PWL), with input constraints. A PWL system is composed of several linear subsystems together with switching rules. Many works

\footnotetext{
* This work was partially supported by CICYT - FEDER projects DPI2006-15390, TIN2007-66523, and by the European Communitys Seventh Framework Programme under project DISC (Grant Agreement n. INFSO-ICT-224498). The work of H.Apaydin-Özkan was supported in part by the Diputacin General de Aragón for one year stay in the Group of Zaragoza; on leave from Faculty of Electrical and Electronics Engineering, Anadolu University, Eskisehir, Turkey. This paper has been written in honor to Prof. L. Recalde, who passed away last December.
}

deal with stability analysis or control law design of such systems (Montagner et al. (2006); Yang et al. (2006); Habets et al. (2006)). However, most of these methods cannot be directly applied to contPNs, because of their particular dynamic input constraints.

Control of contPNs with infinite server semantics has already been considered in the literature. In Mahulea et al. (2008b) it is shown that, the optimal steady state control problem of timed contPN system can be solved by means of Linear Programming Problem (LPP) when all transitions are assumed to be controllable. The transitory control problem is also solved by means of implicit and explicit MPC control strategy (Mahulea et al. (2008a)). A Lyapunov-function-based dynamic control algorithm was proposed in (Xu et al. (2008)), which can ensure global convergence of both system states and input signals. In contrast to the method in this last reference, the technique proposed here for the computation of control law for direct linear trajectory is based on linear programming instead of nonlinear programming. Although the complexity of this new approach is significantly lower, i.e., it can be solved in polynomial time, the time of the obtained trajectory is very similar in general. Moreover, the method in (Xu et al. (2008)) requires solving a BiLinear Programming Problem (BLP) for the computation of intermediate states as our method requires. Refinement of the trajectory by means of intermediate states is carried out by a recursive algorithm which computes new intermediate states until the time can not be improved significantly.

In this work, we design a control strategy which aims at driving the system from an initial state to a target one by minizing the time. The proposed strategy computes first the control actions to drive the system to the target state through a straight line. Such control actions are the result of solving a LPP. Then, as in Xu et al. (2008), in order to obtain faster trajectories, intermediate states, not necessarily on the line connecting the initial and the target marking, are computed by means of a BLP. Moreover, 
an algorithm that recursively refines the initially obtained trajectory is given.

This paper is structured as follows. Section 2 briefly introduces the required concepts of contPN systems and introduces the formulation of applied control. A LPP based method to obtain linear trajectories in order to reach target marking, $\boldsymbol{m}_{f}$, from initial marking $\boldsymbol{m}_{0}$ is given in Section 3. In Section 4, the heuristics for minimizing time by means of intermediate states is considered. Finally, some conclusions and future directions are drawn in Section 5 .

\section{CONTINUOUS PETRI NETS}

We assume that the reader is familiar with discrete PNs. In contPN systems, the firing is not restricted to the natural numbers but to the nonnegative real numbers.

\subsection{Timed Continuous Petri nets}

Definition 1. A continuous PN system is a pair $\left\langle\mathcal{N}, \boldsymbol{m}_{0}\right\rangle$ where $\mathcal{N}=\langle P, T, P r e, P o s t\rangle$ is the net structure with the set of places $P$, the set of transitions $T$, pre and post matrices Pre, Post $\in \mathbb{R}_{\geq 0}^{|P| \times|T|}$ and $\boldsymbol{m}_{0} \in \mathbb{R}_{\geq 0}^{|P|}$ is the initial state.

Let $p_{i}, i=1, \ldots,|P|$ and $t_{j}, j=1, \ldots,|T|$ denote the places and transitions. For a place $p_{i} \in P$ and a transition $t_{j} \in T$, Pre $_{i j}$ and Post $_{i j}$ represent the weights of the arcs from $p_{i}$ to $t_{j}$ and from $t_{j}$ to $p_{i}$, respectively. Each place $p_{i}$ has a marking denoted by $m_{i} \in \mathbb{R}_{\geq 0}$. The vector of all token loads is called state or marking, and is denoted by $\boldsymbol{m} \in \mathbb{R}_{\geq 0}^{|P|}$. For every node $v \in P \cup T$, the sets of its input and output nodes are denoted as $\bullet$ and $v^{\bullet}$, respectively.

A transition $t_{j} \in T$ is enabled at $\boldsymbol{m}$ iff $\forall p_{i} \in t_{j}, m_{i}>0$ and its enabling degree is given by

$$
\operatorname{enab}\left(t_{j}, \boldsymbol{m}\right)=\min _{p_{i} \in t_{j}}\left\{\frac{m_{i}}{\operatorname{Pre}_{i j}}\right\}
$$

which represents the maximum amount in which $t_{j}$ can fire. An enabled transition $t_{j}$ can fire in any real amount $\alpha$, with $0<\alpha \leq \operatorname{enab}\left(t_{j}, \boldsymbol{m}\right)$ leading to a new state $\boldsymbol{m}^{\prime}=\boldsymbol{m}+\alpha \cdot \boldsymbol{C}_{\cdot j}$ where $\boldsymbol{C}=\boldsymbol{P o s t}-\boldsymbol{P r} \boldsymbol{e}$ is the token flow matrix and $\boldsymbol{C}_{\cdot j}$ is its $j^{\text {th }}$ column. If $\boldsymbol{m}$ is reachable from $\boldsymbol{m}_{0}$ through a finite sequence $\sigma$, the state (or fundamental) equation is satisfied: $\boldsymbol{m}=\boldsymbol{m}_{0}+\boldsymbol{C} \cdot \boldsymbol{\sigma}$, where $\boldsymbol{\sigma} \in \mathbb{R}_{\geq 0}^{|T|}$ is the firing count vector, i.e., $\sigma_{j}$ is the cumulative amount of firings of $t_{j}$ in the sequence $\sigma$.

Definition 2. A contPN system $\left\langle\mathcal{N}, \boldsymbol{\lambda}, \boldsymbol{m}_{0}\right\rangle$ is a continuous PN system $\left\langle\mathcal{N}, \boldsymbol{m}_{0}\right\rangle$ together with a vector $\boldsymbol{\lambda} \in \mathbb{R}_{>0}^{|T|}$ where $\lambda_{j}$ is the firing rate of transition $t_{j}$.

The state equation has an explicit dependence on time, denoted by $\tau: \boldsymbol{m}(\tau)=\boldsymbol{m}_{0}+\boldsymbol{C} \cdot \boldsymbol{\sigma}(\tau)$ which through time differentiation becomes $\dot{\boldsymbol{m}}(\tau)=\boldsymbol{C} \cdot \dot{\boldsymbol{\sigma}}(\tau)$. The derivative of the firing sequence $\boldsymbol{f}(\tau)=\dot{\boldsymbol{\sigma}}(\tau)$ is called the firing flow. Depending on how the flow is defined, many firing semantics appear, being the most used ones infinite and finite server semantics. This paper deals with infinite server semantics for which the flow of a transition $t_{j}$ is defined as:

$$
f_{j}(\tau)=\lambda_{j} \cdot \operatorname{enab}\left(t_{j}, \boldsymbol{m}(\tau)\right)=\lambda_{j} \cdot \min _{p_{i} \in t_{j}}\left\{\frac{m_{i}(\tau)}{\text { Pre }_{i j}}\right\}
$$

Since the flow of a transition depends on its enabling degree which is based on the minimum function, a timed contPN with infinite server semantics is a PWL system with polyhedral regions and everywhere continuous vector field. The state space (or reachability set) $\mathcal{R}$ of a contPN system can be divided into regions ${ }^{1}$ as follows: $\mathcal{R}=\mathcal{R}^{1} \cup$ $\ldots \cup \mathcal{R}^{\gamma}$ where $\gamma \leq \prod_{j=1}^{|T|}\left|\bullet t_{j}\right|$. Intuitively, each $\mathcal{R}^{z}$ denotes a region where the flow is limited by the same subset of places (one for each transition). More formally, two markings $m_{a}$ and $m_{b}$ belong to the same region $\mathcal{R}^{z}$ iff $\left[\forall t \in T\right.$ and $\forall p_{u}, p_{v} \in{ }^{\bullet} t$ it holds that $m_{a}\left(p_{u}\right) \leq$ $\left.m_{a}\left(p_{v}\right) \leftrightarrow m_{b}\left(p_{u}\right) \leq m_{b}\left(p_{v}\right)\right]$.

For a given $\mathcal{R}^{z}$, we can define the constraint matrix $\boldsymbol{\Pi}^{z} \in \mathbb{R}_{\geq 0}^{|P|}$ as follows. Let $\boldsymbol{m}$ be an interior point of $\mathcal{R}^{z}$, then $\Pi^{z}$ is defined as:

$$
\boldsymbol{\Pi}_{j i}^{z}=\left\{\begin{array}{ll}
\frac{1}{\text { Pre }_{i j}}, & \text { if } \frac{m_{i}}{\text { Pre }_{i j}}=\min _{p_{h} \in \bullet_{j}}\left\{\frac{m_{h}}{\text { Pre }_{h j}}\right.
\end{array}\right\}
$$

If marking $\boldsymbol{m}$ belongs to $\mathcal{R}^{z}$, we denote $\boldsymbol{\Pi}(\boldsymbol{m})=\boldsymbol{\Pi}^{z}$ the corresponding constraint matrix (if $\boldsymbol{m}$ is a border point and belongs to several regions, $\boldsymbol{\Pi}(\boldsymbol{m})$ is the constraint matrix of any of these regions). In the constraint matrix, each row $j=1,2 \ldots,|T|$ has only one non-null element in the position $i$ that corresponds to the place $p_{i}$ that restricts the flow of transition $t_{j}$. Furthermore, the firing rate of transitions can also be represented by a diagonal matrix $\boldsymbol{\Lambda}=\operatorname{diag}\left\{\lambda_{1}, \ldots . \lambda_{|T|}\right\}$. Using this notation, the nonlinear flow of the transitions at a given state $\boldsymbol{m}$ can be written as:

$$
\boldsymbol{f}=\Lambda \cdot \Pi(\boldsymbol{m}) \cdot \boldsymbol{m}
$$

Example 3. Let us consider the PN in Fig. 1. Assume $\boldsymbol{\lambda}=\left[\begin{array}{llll}4 & 1 & 3 & 1\end{array}\right]^{T}, \boldsymbol{m}_{0}=\left[\begin{array}{llllllll}7.5 & 4.5 & 4 & 2 & 1.5 & 5 & 4 & 2.5\end{array}\right]^{T}$ and $\boldsymbol{m}_{f}=\left[\begin{array}{llllllll}7 & 5 & 5 & 1 & 1 & 4 & 5 & 3\end{array}\right]^{T}$.

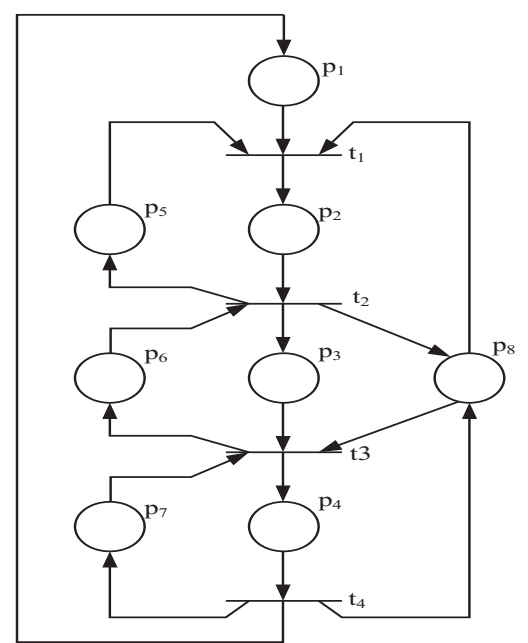

Fig. 1. A PN taken from (Zhou et al. (1990))

1 These regions are disjoint except possibly on the borders. 
Independently of $\boldsymbol{m}_{0}$, the system dynamics is described as follows:

$$
\begin{aligned}
\dot{m}_{1}= & f_{4}-f_{1}=\lambda_{4} \cdot m_{4}-\lambda_{1} \cdot \min \left\{m_{5}, m_{1}, m_{8}\right\} \\
\dot{m}_{2}= & f_{1}-f_{2}=\lambda_{1} \cdot \min \left\{m_{5}, m_{1}, m_{8}\right\}-\lambda_{2} \cdot \min \left\{m_{2}, m_{6}\right\} \\
\dot{m}_{3}= & f_{2}-f_{3}=\lambda_{2} \cdot \min \left\{m_{2}, m_{6}\right\}-\lambda_{3} \cdot \min \left\{m_{3}, m_{7}, m_{8}\right\} \\
\dot{m}_{4}= & f_{3}-f_{4}=\lambda_{3} \cdot \min \left\{m_{3}, m_{7}, m_{8}\right\}-\lambda_{4} \cdot m_{4} \\
\dot{m}_{5}= & f_{2}-f_{1}=\lambda_{2} \cdot \min \left\{m_{2}, m_{6}\right\}-\lambda_{1} \cdot \min \left\{m_{5}, m_{1}, m_{8}\right\} \\
\dot{m}_{6}= & f_{3}-f_{2}=\lambda_{3} \cdot \min \left\{m_{3}, m_{7}, m_{8}\right\}-\lambda_{2} \cdot \min \left\{m_{2}, m_{6}\right\} \\
\dot{m}_{7}= & f_{4}-f_{3}=\lambda_{4} \cdot m_{4}-\lambda_{3} \cdot \min \left\{m_{3}, m_{7}, m_{8}\right\} \\
\dot{m}_{8}= & f_{2}+f_{4}-f_{1}-f_{3}=\lambda_{2} \cdot \min \left\{m_{2}, m_{6}\right\}+\lambda_{4} \cdot m_{4} \\
& -\lambda_{1} \cdot \min \left\{m_{5}, m_{1}, m_{8}\right\}-\lambda_{3} \cdot \min \left\{m_{3}, m_{7}, m_{8}\right\}
\end{aligned}
$$

Note that $\boldsymbol{m}_{0}$ and $\boldsymbol{m}_{f}$ are in different regions: $\boldsymbol{m}_{0} \in \mathcal{R}^{1}$ : $\left\{\boldsymbol{m} \mid m_{5} \leq m_{1}, m_{5} \leq m_{8}, m_{8} \leq m_{7}, m_{8} \leq m_{3}, m_{2} \leq\right.$ $\left.m_{6}\right\}$ and $\boldsymbol{m}_{f} \in \mathcal{R}^{2}:\left\{\boldsymbol{m} \mid m_{5} \leq m_{1}, m_{5} \leq m_{8}, m_{8} \leq\right.$ $\left.m_{7}, m_{8} \leq m_{3}, m_{6} \leq m_{2}\right\}$.

For example, the system dynamics for $\mathcal{R}^{1}$ are:

$$
\left\{\begin{array}{l}
\dot{m}_{1}=m_{4}-4 \cdot m_{5} \\
\dot{m}_{2}=4 \cdot m_{5}-m_{2} \\
\dot{m}_{3}=m_{2}-3 \cdot m_{8} \\
\dot{m}_{4}=3 \cdot m_{8}-m_{4} \\
\dot{m}_{5}=m_{2}-4 \cdot m_{5} \\
\dot{m}_{6}=3 \cdot m_{8}-m_{2} \\
\dot{m}_{7}=m_{4}-m_{8} \\
\dot{m}_{8}=m_{2}+m_{4}-4 \cdot m_{5}+3 \cdot m_{8}
\end{array}\right.
$$

\subsection{Controlled Timed Continuous Petri Nets}

In this section we consider contPN systems subject to external control actions, and assume that the only admissible control law consists in slowing-down the firing speed of transitions (Silva and Recalde (2004)). If a transition can be controlled (its flow can be reduced or even stopped), we will say that it is a controllable transition (Mahulea et al. (2008b)).

Definition 4. The controlled flow, $\boldsymbol{w}$, of a timed contPN is defined as $\boldsymbol{w}(\tau)=\boldsymbol{f}(\tau)-\boldsymbol{u}(\tau)$, with $0 \leq \boldsymbol{u}(\tau) \leq \boldsymbol{f}(\tau)$, where $f$ is the flow of the uncontrolled system, i.e., defined as in (1), and $\boldsymbol{u}$ is the control action.

Therefore, the control input $\boldsymbol{u}$ is dynamically upper bounded by the flow $f$ of the corresponding unforced system. Under these conditions, the overall behaviour of the system in which all transitions are controllable is ruled by the following system:

$$
\begin{aligned}
& \dot{m}=C \cdot[f-u]=C \cdot w \\
& 0 \leq u \leq f
\end{aligned}
$$

The constraint $0 \leq \boldsymbol{u} \leq \boldsymbol{f}$ can be rewritten as $0 \leq \boldsymbol{f}-\boldsymbol{u} \leq \boldsymbol{f}$. Defining $\boldsymbol{w}=\boldsymbol{f}-\boldsymbol{u}$ and using (3), the constraint can be expressed as:

$$
0 \leq \boldsymbol{w} \leq \boldsymbol{\Lambda} \cdot \boldsymbol{\Pi}(\boldsymbol{m}) \cdot \boldsymbol{m}
$$

The following sections show how to compute a control action $\boldsymbol{u}$ that drives the system from the initial marking $\boldsymbol{m}_{0}$ to a desired target marking $\boldsymbol{m}_{f}$. Section 3 describes a method to obtain a linear trajectory. Section 4 makes use of such a method to compute piecewise linear trajectories in order to improve the time to reach $\boldsymbol{m}_{f}$. Notice that in order to be reachable, $\boldsymbol{m}_{f}$ necessarily satisfies the state equation, i.e., $\boldsymbol{m}_{f}=\boldsymbol{m}_{0}+\boldsymbol{C} \cdot \boldsymbol{\sigma}$. Our procedure consists of assigning constant controlled flow $\boldsymbol{w}$ that satisfies dynamic upper bounds. We assume that $\boldsymbol{m}_{0}$ and $\boldsymbol{m}_{f}$ are interior points of the reachability space, i.e., the markings are strictly positive. The assumption that $\boldsymbol{m}_{0}$ is positive ensures that the system can move at $\tau=0$ in the direction of $\boldsymbol{m}_{f}$; the assumption that $\boldsymbol{m}_{f}$ is positive ensures that $\boldsymbol{m}_{f}$ can be reached (Júlvez et al. (2003)) in finite time (Mahulea et al. (2008b)).

\section{COMPUTATION OF LINEAR TRAJECTORIES}

In this section, we will distinguish two cases: (A) $\boldsymbol{m}_{0}$ and $\boldsymbol{m}_{f}$ are in the same region and (B) they are in different regions. In this last case the crossing points of the straight line and borders must be considered.

(A) $\boldsymbol{m}_{0}$ and $\boldsymbol{m}_{f}$ are in the same region $\mathcal{R}^{z}$. Then all the states on the straight line connecting them are also interior points of $\mathcal{R}^{z}$ since all the regions are convex. The following LPP computes the correspondig control law, where $\boldsymbol{x}=\boldsymbol{w} \cdot \tau_{f}$,

$$
\begin{aligned}
\min & \tau_{f} \\
& \boldsymbol{m}_{f}=\boldsymbol{m}_{0}+\boldsymbol{C} \cdot \boldsymbol{x} \\
0 \leq x_{j} \leq \lambda_{j} \cdot \Pi_{j i}^{z} \cdot \min \left\{m_{0 i}, m_{f_{i}}\right\} \cdot \tau_{f}, & (a) \\
& \forall j \in\{1, . .,|T|\} \text { where } i \text { satisfies } \Pi_{j i}^{z} \neq 0 \quad(b)
\end{aligned}
$$

The equations correspond to: (a) the time dependent equation of the straight line connecting $\boldsymbol{m}_{0}$ to $\boldsymbol{m}_{f}$, (b) flow constraints in (7). Notice that $(8)(\mathrm{b})$ is a linear constraint because $m_{0_{i}}$ and $m_{f_{i}}$ are known.

(B) $\boldsymbol{m}_{0}$ and $\boldsymbol{m}_{f}$ are in different regions. The line connecting $\boldsymbol{m}_{0}$ and $\boldsymbol{m}_{f}$ can be divided in several segments, each one starting in a border (or in $\boldsymbol{m}_{0}$ for the first segment) and ending in a border (or in $\boldsymbol{m}_{f}$ for the last one). Then LPP (8) is applied on each of these segments.

Algorithm 1 computes the total time $\tau_{f}$ by using LPP in (8) for the linear trajectory $\ell$ from $\boldsymbol{m}_{0}$ to $\boldsymbol{m}_{f}$ which crosses $n \in\{1,2, . ., \gamma\}$ borders. In this algorithm, $\boldsymbol{m}_{c}^{i}$ stands for state at the intersection of $\ell$ and $i^{\text {th }}$ crossed border (starting from $\boldsymbol{m}_{0}$ ) $i \in\{1,2, . ., n\} . \boldsymbol{m}_{c}^{0}$ and $\boldsymbol{m}_{c}^{n+1}$ denote $\boldsymbol{m}_{0}$ and $\boldsymbol{m}_{f}$, respectively. $\boldsymbol{w}^{i}$ is the flow obtained from the state $\boldsymbol{m}_{c}^{i}$ to the state $\boldsymbol{m}_{c}^{i+1}$. Note that, if $\boldsymbol{\ell}$ does not cross any border, then $n=0$, that is $\boldsymbol{m}_{c}^{0}=\boldsymbol{m}_{0}$ and $\boldsymbol{m}_{c}^{1}=\boldsymbol{m}_{f}$.

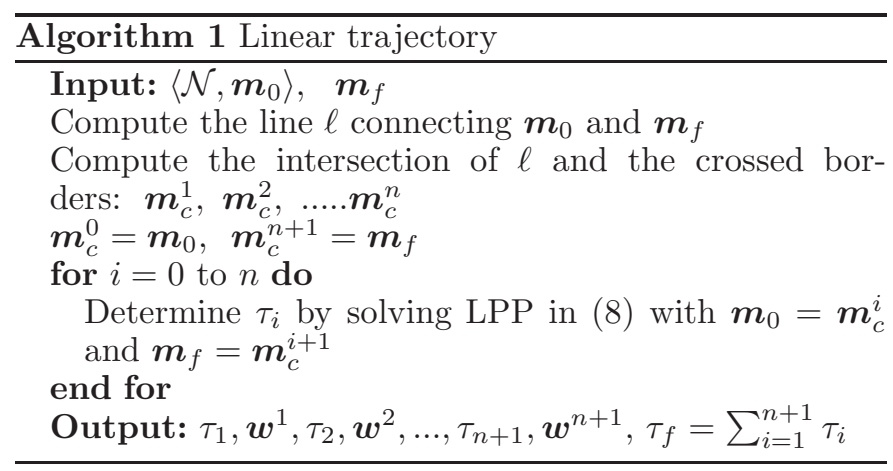

Proposition 5. Let $\left\langle\mathcal{N}, \boldsymbol{\lambda}, \boldsymbol{m}_{0}\right\rangle$ be a contPN system with $\boldsymbol{m}_{0}>0$. If $\boldsymbol{m}_{f}$ belongs to $\mathcal{R}$ and $\boldsymbol{m}_{f}>0$ :

- $\operatorname{LPP}(8)$ is feasible

- The control action given by Algorithm 1 ensures that $\boldsymbol{m}_{f}$ is reached in finite time.

Proof. Since $\boldsymbol{m}_{f}$ is a reachable marking, then there exists $\boldsymbol{x}$ such that the state equation is satisfied 8(a). 
By taking $\tau_{f}$ sufficiently large $8(\mathrm{~b})$ can be satisfied since $\lambda_{j} \cdot \Pi_{j i}^{z} \cdot \min \left\{m_{0 i}, m_{f}\right\}>0$. Then, by (Júlvez et al. (2003)) the linear trajectory from $\boldsymbol{m}_{0}$ to $\boldsymbol{m}_{f}$ can be followed by the system since $\boldsymbol{m}_{0}>0$ and $\boldsymbol{m}_{f}>0$, i.e. , all intermediate states are not spurious. Moreover, since $\boldsymbol{m}_{f}>0$, by (Mahulea et al. (2008b)) $\boldsymbol{m}_{f}$ can be reached in finite time.

Example 6. Let us consider the control law design for the contPN in Ex. 3. Assume the same $\boldsymbol{\lambda}, \boldsymbol{m}_{0}$ and $\boldsymbol{m}_{f}$. Note that the line $\ell$ connecting $\boldsymbol{m}_{0}$ and $\boldsymbol{m}_{f}$ crosses only one border: $m_{2}=m_{6}$ and the crossing point is calculated as: $\boldsymbol{m}_{c}^{1}=\left[\begin{array}{lllll}7.33 & 4.674 .331 .671 .334 .674 .33 & 2.67\end{array}\right]^{T}, \boldsymbol{m}_{c}^{0}=\boldsymbol{m}_{0}$, $\boldsymbol{m}_{c}^{2}=\boldsymbol{m}_{f}$. The trajectory is illustrated in Fig.2. According

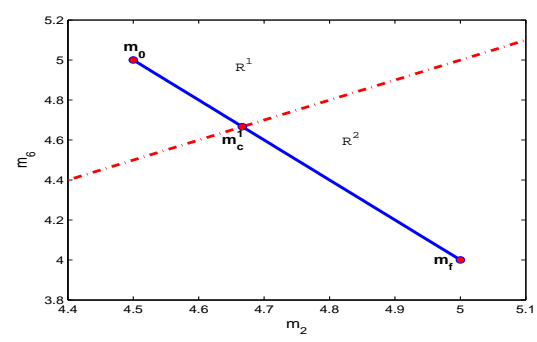

Fig. 2. Trajectory for Ex. 6

to Algorithm 1, first LPP (8) with $\boldsymbol{m}_{0}=\boldsymbol{m}_{c}^{0}$ and $\boldsymbol{m}_{f}=$ $\boldsymbol{m}_{c}^{1}$ is solved. Its constraints are:

$$
\begin{aligned}
7.33 & =x_{4}-x_{1}+7.5 \\
4.67 & =x_{1}-x_{2}+4.5 \\
4.33 & =x_{2}-x_{3}+4 \\
1.67 & =x_{3}-x_{4}+2 \\
1.33 & =x_{2}-x_{1}+1.5 \\
4.67 & =x_{3}-x_{2}+5 \\
4.33 & =x_{4}-x_{3}+4 \\
2.67 & =x_{2}+x_{4}-x_{1}-x_{3}+2.5 \\
0 & \leq x_{1} \leq 4 \cdot 1.33 \cdot \tau_{f} \\
0 & \leq x_{2} \leq 4.5 \cdot \tau_{f} \\
0 & \leq x_{3} \leq 3 \cdot 2.5 \tau_{f} \\
0 & \leq x_{4} \leq 1.66 \cdot \tau_{f}
\end{aligned}
$$

The optimal solution is $\tau_{f}=0.2$ t.u. and $w_{1}=2.49$, $w_{2}=1.67, w_{3}=0, w_{4}=1.67$. In the second case, i.e., $\boldsymbol{m}_{0}=\boldsymbol{m}_{c}^{1}$ and $\boldsymbol{m}_{f}=\boldsymbol{m}_{c}^{2}$ :

$$
\begin{aligned}
& 7=x_{4}-x_{1}+7.33 \\
& 5=x_{1}-x_{2}+4.67 \\
& 5=x_{2}-x_{3}+4.33 \\
& 1=x_{3}-x_{4}+1.67 \\
& 1=x_{2}-x_{1}+1.33 \\
& 4=x_{3}-x_{2}+4.67 \\
& 5=x_{4}-x_{3}+4.33 \\
& 3=x_{2}+x_{4}-x_{1}-x_{3}+2.67 \\
& 0 \leq x_{1} \leq 4 \cdot \tau_{f} \\
& 0 \leq x_{2} \leq 4 \cdot \tau_{f} \\
& 0 \leq x_{3} \leq 3 \cdot 2.67 \cdot \tau_{f} \\
& 0 \leq x_{4} \leq \tau_{f}
\end{aligned}
$$

The optimal solution is $\tau_{f}=0.67$ t.u. and $w_{1}=1.5$, $w_{2}=1, w_{3}=0, w_{4}=1$. The total time to go from $\boldsymbol{m}_{0}$ to $\boldsymbol{m}_{f}$ through the line $\ell$ is $\tau_{\text {total }}=0.2+0.67=0.87$ t.u. And the control is obtained as:

$$
\boldsymbol{u}(\tau)=\left\{\begin{array}{r}
{\left[\begin{array}{r}
4 \cdot m_{5}(\tau)-2.49 \\
m_{2}(\tau)-1.67 \\
3 \cdot m_{8}(\tau) \\
m_{4}(\tau)-1.67
\end{array}\right], \text { if } 0 \leq \tau \leq 0.2} \\
{\left[\begin{array}{r}
4 \cdot m_{5}(\tau)-1.5 \\
m_{2}(\tau)-1 \\
3 \cdot m_{8}(\tau) \\
m_{4}(\tau)-1
\end{array}\right], \text { if } 0.2<\tau \leq 0.87}
\end{array}\right.
$$

Fig. 3(a) illustrates the convergence of the marking $m_{1}$ under the designed control law, while Fig. 3(b) shows the control signal $u_{1}, w_{1}$ and their upper bound.

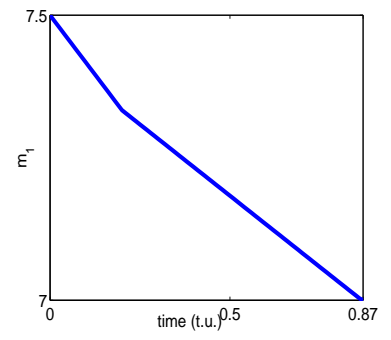

(a)

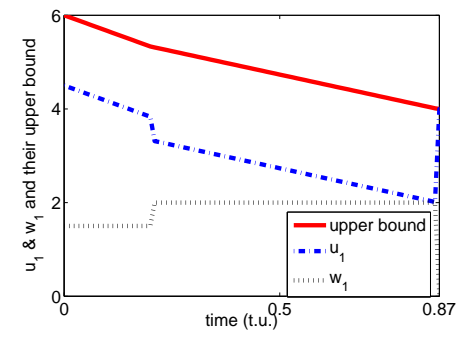

(b)
Fig. 3. Evolution of (a) $m_{1}$ and (b) $u_{1}, w_{1}$ and and their upper bound for Ex. 6

\section{A HEURISTICS FOR MINIMUM TIME CONTROL}

This section illustrates how to compose a piecewise linear trajectory by introducing intermediate states in order to improve the time duration to reach $\boldsymbol{m}_{f}$.

The designed control in the previous subsection takes the system from $\boldsymbol{m}_{0}$ to $\boldsymbol{m}_{f}$ through a linear trajectory by minimizing the time duration. In some cases, i.e., when initial or final state have a small value, this may limit the speed of the response. In order to improve the time spent to move from $\boldsymbol{m}_{0}$ to $\boldsymbol{m}_{f}$, intermediate states denoted by $\boldsymbol{m}^{k}$ where $k \in\{1,2 \ldots s\}$, that do not necessarily lie on the line from $\boldsymbol{m}_{0}$ to $\boldsymbol{m}_{f}$, are computed. The new trajectory is obtained as the union of $s+1$ segments: $\boldsymbol{m}_{0} \rightarrow \boldsymbol{m}^{1} \rightarrow \boldsymbol{m}^{2} \rightarrow \ldots \rightarrow \boldsymbol{m}^{s} \rightarrow \boldsymbol{m}_{f}$. In this work we consider the intermediate states in the range:

$$
\begin{gathered}
\mathcal{R}^{I}=\left\{\boldsymbol{m} \mid m_{i} \leq \max \left\{m_{f_{i}}, m_{0_{i}}\right\}, \quad m_{i} \geq \min \left\{m_{f_{i}}, m_{0_{i}}\right\},\right. \\
i \in\{1,2, . .,|P|\}\},
\end{gathered}
$$

Because $\boldsymbol{m}_{0}, \boldsymbol{m}_{f}>0$, all $\boldsymbol{m} \in \mathcal{R}^{I}$ are positive and reachable (Júlvez et al. (2003)). We will describe how to compute intermediate states that lie on the borders and the interior of regions, separately.

\subsection{Intermediate states on the borders}

Let the line from $\boldsymbol{m}_{0}$ to $\boldsymbol{m}_{f}$ cross $s$ borders, and pass through $s+1$ different regions: $\mathcal{R}^{1}, \mathcal{R}^{2}, \ldots, \mathcal{R}^{s+1}$ with corresponding constraint matrices $\Pi^{1}, \Pi^{2} \ldots \Pi^{s+1}$. We assign constant flows for each transitions during each line segments and the intermediate states on each border: $\boldsymbol{m}^{1}, \boldsymbol{m}^{2} \ldots \boldsymbol{m}^{s}$. Under these assumptions, the following 
BLP with $\boldsymbol{m}^{0}=\boldsymbol{m}_{0}$ and $\boldsymbol{m}^{s+1}=\boldsymbol{m}_{f}$ and with the variables $\boldsymbol{m}^{1}, \boldsymbol{m}^{2}, \ldots, \boldsymbol{m}^{s-1}, \boldsymbol{m}^{s}, \tau_{k}, \quad \boldsymbol{x}^{k}=\boldsymbol{w}^{k} \cdot \tau_{k}$, $k \in\{1, \ldots s\}$ obtains the intermediate states that yield a minimum time trajectory:

$$
\begin{aligned}
& \min \sum_{k=1}^{s+1} \tau_{k} \\
& \boldsymbol{m}^{k+1}=\boldsymbol{m}^{k}+\boldsymbol{C} \cdot \boldsymbol{x}^{k+1}, \quad k \in\{0,1,2, . ., s\} \\
& \left(\Pi^{k}-\boldsymbol{\Pi}^{k+1}\right) \cdot \boldsymbol{m}^{k}=0, \quad k \in\{1,2, . ., s\} \\
& m_{i}^{k} \leq m_{i}^{k+1} \text { if } \quad \begin{array}{l}
m_{0_{i}} \leq m_{f_{i}} \\
i \in\{1,2 . .|P|\}, \quad k \in\{0,1,2, . ., s\}
\end{array} \\
& m_{i}^{k} \geq m_{i}^{k+1} \quad \text { if } \quad \begin{array}{l}
m_{0_{i}} \geq m_{f_{i}} \\
i \in\{1,2 . .|P|\}, \quad k \in\{0,1,2, . ., s\}
\end{array} \\
& 0 \leq x_{j}^{k} \leq \lambda_{j} \cdot \Pi_{j i}^{k} \cdot \min \left\{m_{i}^{k-1}, m_{i}^{k}\right\} \cdot \tau_{k}, \\
& \text { with } p_{i} \text { st. } \Pi_{j i}^{k} \neq 0, j \in\{1,2 . . . T \mid\}, \quad k \in\{1,2 . . s\}
\end{aligned}
$$

The constraints correspond to (a) the time dependent equation of the connecting $\boldsymbol{m}^{k}$ to $\boldsymbol{m}^{k+1}: \boldsymbol{m}^{k+1}=\boldsymbol{m}^{k}+\boldsymbol{C}$. $\boldsymbol{w}^{k+1} \cdot \tau_{f}$ and $\boldsymbol{m}^{k}$ is a reachable marking; (b) $\boldsymbol{m}^{k}$ is on the crossed border; (c \& d) $\boldsymbol{m}^{k}$ is contained in the hypercube defined by the corners $\boldsymbol{m}^{k-1}$ and $\boldsymbol{m}^{k+1}$; (e) the constraints on the controlled flow.

\subsection{Interior intermediate states}

In the case that $\boldsymbol{m}_{0}$ and $\boldsymbol{m}_{f}$ are in the same region $\mathcal{R}^{z}$ a recursive method can be used to determine intermediate states. The same recursive method can also be applied to find additional intermediate states between $\boldsymbol{m}^{k}$ and $\boldsymbol{m}^{k+1}$, since they are on the borders of the same region. In this method we keep computing intermediate states until the time cannot be improved by a considerable amount. In this case, the BLP that will be solved in each step can be simplified to the following problem where $\boldsymbol{m}_{0}$ and $\boldsymbol{m}_{f}$ are known; $\tau_{1}, \tau_{2}, \boldsymbol{m}^{d}, \boldsymbol{x}^{1}=\boldsymbol{w}^{1} \cdot \tau_{1}, \boldsymbol{x}^{2}=\boldsymbol{w}^{2} \cdot \tau_{2}$ are variables: $\min \tau_{1}+\tau_{2}$

$$
\begin{aligned}
& \boldsymbol{m}^{d}=\boldsymbol{m}_{0}+\boldsymbol{C} \cdot \boldsymbol{x}^{1} \\
& \boldsymbol{m}_{f}=\boldsymbol{m}^{d}+\boldsymbol{C} \cdot \boldsymbol{x}^{2}, \\
& \boldsymbol{m}^{d}=\boldsymbol{m}_{0}+\boldsymbol{C} \cdot \boldsymbol{\sigma}, \quad \boldsymbol{m}^{d}, \boldsymbol{\sigma} \geq 0 \\
& \min \left\{m_{f_{i}}, m_{0_{i}}\right\} \leq m_{i}^{d} \leq \max \left\{m_{f_{i}}, m_{0_{i}}\right\}, \\
& \forall i \in\{1,2 \ldots|P|\} \quad(c) \\
& 0 \leq x_{j}^{1} \leq \lambda_{j} \cdot \Pi_{j i}^{z} \cdot \min \left\{m_{0_{i}}, m_{i}^{d}\right\} \cdot \tau_{1}, \\
& \text { with } i \text { st. } \Pi_{j i}^{k} \neq 0, \quad j \in\{1,2 \ldots|T|\} \\
& 0 \leq x_{j}^{2} \leq \lambda_{j} \cdot \Pi_{j i}^{z} \cdot \min \left\{m_{i}^{d}, m_{f_{i}}\right\} \cdot \tau_{2}, \\
& \text { with } i \text { st. } \Pi_{j i}^{k} \neq 0, \quad j \in\{1,2 \ldots|T|\} \quad(d)
\end{aligned}
$$

Algorithm 2 expresses the recursive method we proposed. It computes new intermediate states until the stopping criterion is satisfied. It is satisfied when the time of the trajectory cannot be decreased more than a given value $\epsilon$. In the algorithm, path is a global variable storing the ordered set of couples of states in the trajectory and the relative times to reach them. The number of states in path is denoted by size (path) and the $i^{\text {th }}$ element of the set path is denoted by path $(i)$.
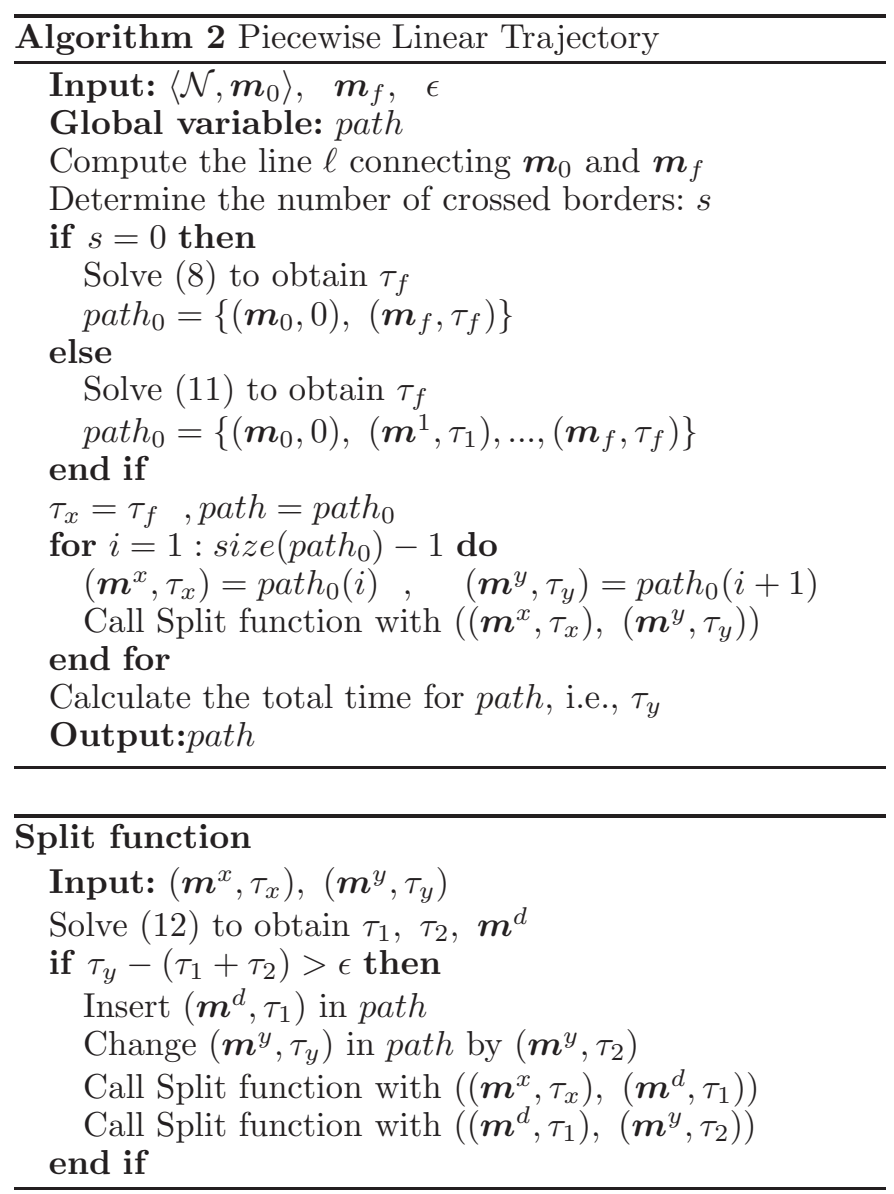

Example \%. Let us consider the contPN system considered in Ex. 6, again. Since the linear trajectory between $\boldsymbol{m}_{0}$ and $\boldsymbol{m}_{f}$ crosses only one border, $s=1$ and solving (11) with $s=1$ yields 0.83 t.u. as a total time. And the new path is $\boldsymbol{m}_{0} \rightarrow \boldsymbol{m}^{1} \rightarrow \boldsymbol{m}_{f}$, where $\boldsymbol{m}^{1}=$ $\left[\begin{array}{llllllll}7.5 & 4.5 & 4.5 & 1.5 & 1.5 & 4.5 & 4.5 & 3\end{array}\right]^{T}$. For an additional intermediate state, say $\boldsymbol{m}^{\prime 1}$, between $\boldsymbol{m}_{0}$ and $\boldsymbol{m}^{1}$, we solve

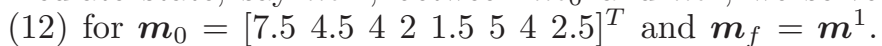
Similarly, for an additional intermediate state between $\boldsymbol{m}^{1}$ and $\boldsymbol{m}_{f}$, say $\boldsymbol{m}^{\prime 2}$, we solve (12) for $\boldsymbol{m}_{0}=\boldsymbol{m}^{1}$ and $\boldsymbol{m}_{f}=\left[\begin{array}{llllllll}7 & 5 & 5 & 1 & 1 & 4 & 5 & 3\end{array}\right]^{T}$. And the new path $\boldsymbol{m}_{0} \rightarrow \boldsymbol{m}^{\prime 1} \rightarrow \boldsymbol{m}^{1} \rightarrow \boldsymbol{m}^{\prime 2} \rightarrow \boldsymbol{m}_{f}$ is obtained, where $\boldsymbol{m}^{\prime 1}=\left[\begin{array}{llllllll}7.5 & 4.5 & 4.26 & 1.73 & 1.5 & 4.73 & 4.26 & 2.76\end{array}\right]^{T}$ and $\boldsymbol{m}^{\boldsymbol{\prime}^{2}}=$

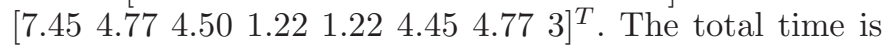
reduced to 0.74 t.u. with the control action $u(\tau)=[4$. $\left.m_{5}(\tau)-1.73 \quad m_{2}(\tau)-1.73 \quad 3 \cdot m_{8}(\tau) m_{4}(\tau)-1.7321\right]^{T}$ for $0 \leq \tau \leq 0.15 ; u(\tau)=\left[4 \cdot m_{5}(\tau)-1.5 m_{2}(\tau)-1.53\right.$. $\left.m_{8}(\tau) m_{4}(\tau)-1.5\right]^{T}$ for $0.15 \leq \tau \leq 0.3 ; u(\tau)=\left[4 \cdot m_{5}(\tau)-\right.$ $\left.1.23 m_{6}(\tau) 3 \cdot m_{8}(\tau) m_{4}(\tau)-1.2247\right]^{T}$ for $0.3 \leq \tau \leq 0.51$;

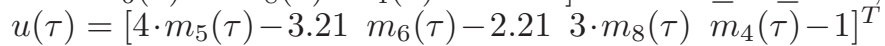
for $0.51 \leq \tau \leq 0.74$. The trajectories for one and three intermediate states are illustrated in Fig. 4(a) and Fig. 4(b), respectively. In these figures dotted line shows the border between $\mathcal{R}^{1}$ and $\mathcal{R}^{2}$. The total time duration for several intermediate states can be found in Table 1 . The experiments were performed by a Matlab program running on a PC with Intel(R) Core(TM)2CPU T5600 @ $1.83 \mathrm{GHz}$, $2.00 \mathrm{~GB}$ of RAM. 


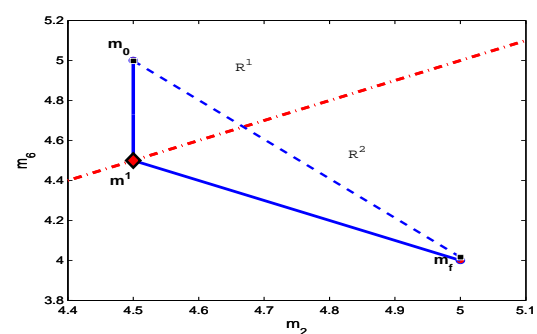

(a)

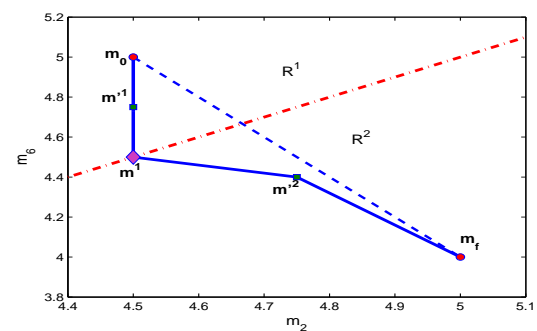

(b)

Fig. 4. Trajectory for (a)1 int. state and (b) 3 int. state for Ex. 7

Table 1. Intermediate States

\begin{tabular}{lccccc} 
Number of int. states & 0 & 1 & 3 & 9 & 13 \\
\hline Total duration (t.u.) & 0.87 & 0.83 & 0.74 & 0.73 & 0.72 \\
CPU time (sec.) & 0.03 & 0.11 & 0.32 & 1.65 & 2.32
\end{tabular}

Example 8. Let us consider the net in Fig. 5 with $\boldsymbol{\lambda}=$ $\left[\begin{array}{lll}3 & 1 & 1\end{array}\right]^{T}$. By using Algorithm 1, the total time to reach $\boldsymbol{m}_{f}=\left[\begin{array}{llll}1 & 10 & 6 & 2\end{array}\right]^{T}$ from $\boldsymbol{m}_{0}=\left[\begin{array}{llll}13 & 3 & 1 & 10\end{array}\right]^{T}$ through a linear trajectory is obtained as 2.43 t.u., with the control $u(\tau)=\left[\begin{array}{lll}3 \cdot m_{4}(\tau)-7 & m_{2}(\tau) \quad m_{3}(\tau)-1\end{array}\right]^{T}$ for $0 \leq \tau \leq$ $0.93, u(\tau)=\left[\begin{array}{lll}3 \cdot m_{4}(\tau)-12 & m_{4}(\tau) & m_{3}(\tau)-1.71\end{array}\right]^{T}$ for $0.93 \leq \tau \leq 1.26, \quad u(\tau)=\left[3 \cdot m_{1}(\tau)-3 \quad m_{2}(\tau) \quad m_{3}(\tau)-\right.$ $0.42]^{\bar{T}}$ for $1.26 \leq \tau \leq 2.43$, while $\boldsymbol{m}_{f}$ was reached in 4.4 t.u. in (Xu et al. (2008)). The piecewise linear trajectory with 4 intermediate states obtained by Algorithm 2 yields 1.38 t.u.

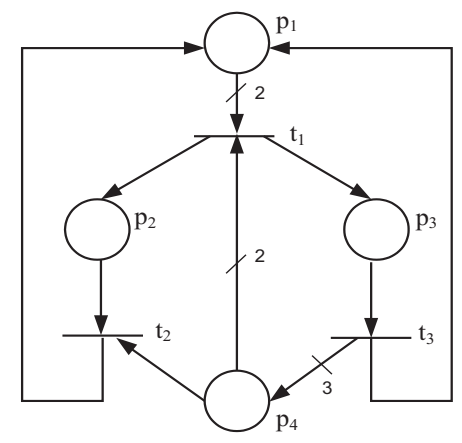

Fig. 5. A PN

\section{CONCLUSION}

The control problem adressed in the paper consists of reaching a target state in minimum time through a piecewise linear trajectory. Besides the piecewise linear dynamics of continuous Petri nets, the control method handles the fact that the input constraints depend on the current marking, i.e., the inputs are dynamically constrained.
The heuristics proposed in this paper computes first a "rough" piecewise linear trajectory that is refined afterwards in those intervals that allow an improvement. The heuristics makes use of BPPs to obtain intermediate states and LPPs to compute linear trajectories. The refinement of the trajectory is achieved recursively .

\section{REFERENCES}

David, R. and Alla, H. (2005). Autonomous and timed continuous Petri nets. Springer, Berlin.

Habets, L., Collins, P., and van Schuppen, J. (2006). Reachability and control synthesis for piecewise-affine hybrid systems on simplices. Automatic Control, IEEE Transactions on, 51(6), 938-948. doi:10.1109/TAC. 2006.876952.

Júlvez, J., Recalde, L., and Silva, M. (2003). On reachability in autonomous continuous Petri net systems. In W. van der Aalst and E. Best (eds.), 24 $4^{\text {th }}$ International Conference on Application and Theory of Petri Nets (ICATPN 2003), volume 2679 of Lecture Notes in Computer Science, 221-240. Springer, Eindhoven, The Netherlands.

Mahulea, C., Giua, A., Recalde, L., Seatzu, C., and Silva, M. (2008a). Optimal model predictive control of timed continuous Petri nets. IEEE Transactions on Automatic Control, 53(7), 1731 - 1735.

Mahulea, C., Ramirez, A., Recalde, L., and M.Silva (2008b). Steady state control reference and token conservation laws in continuous Petri net systems. IEEE Transactions on Automation Science and Engineering, $5(2), 307-320$.

Mahulea, C., Recalde, L., and Silva, M. (2009). Basic Server Semantics and Performance Monotonicity of Continuous Petri Nets. Discrete Event Dynamic Systems: Theory and Applications, 19(2), $189-212$.

Montagner, V.F., Leite, V., Oliveira, R., and Peres, P. (2006). State feedback control of switched linear systems: An LMI approach. Journal of Computational and Applied Mathematics, 94(2), 192-206.

Silva, M. and Recalde, L. (2004). On fluidification of Petri net models: from discrete to hybrid and continuous models. Annual Reviews in Control, 28(2), 253-266.

Xu, J., Recalde, L., and Silva, M. (2008). Tracking control of timed continuous Petri net systems under infinite servers semantics. In 17 th World Congress of IFAC, 3192-3197. Seoul, Korea.

Yang, H., Xie, G., Chu, T., and Wang, L. (2006). Commuting and stable feedback design for switched linear systems. Journal of Computational and Applied Mathematics, 94(2), 192-206.

Zhou, M., DiCesare, F., and Guo, D. (1990). Modeling and performance analysis of a resource-sharing manufacturing system using stochastic Petri nets. In Fifth IEEE Symp. on Intelligent Control, 1005-1010. Phildephia, PA. 